\title{
A case for non-surgical management of splenic abscess in the setting of salmonella typhimurium bacteremia
}

\begin{abstract}
Background: Non-typhoidal salmonella (NTS) infections typically present with symptoms of gastroenteritis. Complications include extra-intestinal infections such as meningitis, osteomyelitis and bacteremia. Here, we report a case of splenic abscess associated with Salmonella typhimurium bacteremia in the setting of uncontrolled type 2 diabetes mellitus (DMII). This represents a less common and potentially fatal complication of NTS.
\end{abstract}

Case presentation: 52year old Caucasian male with a past medical history of uncontrolled DMII, hypertension, and hypercholesterolemia, presented with 2 weeks of flu-like symptoms and left lower back discomfort. He denied any symptoms of nausea, vomiting, or diarrhea. He had no foreign travel. Labs were significant for leucocytosis, elevated CRP, mild transaminitis, and hemoglobin A c of $12.2 \%$. He tested negative for HIV and hepatitis A, B and C. CT-scan of the abdomen and pelvis showed splenomegaly $(14 \mathrm{~cm})$ with a gas and fluid containing collection $(6.2$ x 9.4 x $5.6 \mathrm{~cm})$ suggestive of splenic abscess. Patient was started on intravenous ceftriaxone daily. Blood cultures grew Salmonella typhimurium. Transesophageal echocardiography was negative for vegetations. A percutaneous drain was placed the following day and his symptoms significantly improved. His leucocytosis has resolved as well. The splenic abscess decreased in size on repeated imaging of the abdomen at day six of antibiotics. Outpatient intravenous ceftriaxone was continued for a total of six weeks, along with optimal glycemic control. A follow up CT scan after five weeks of treatment showed near resolution of the splenic abscess. Follow-up blood cultures were negative in addition to significant improvement in his symptoms.

Conclusion: In patients with comorbidities including DMII or other immunocompromised status, NTS should be considered as a causal agent for splenic abscess. Early identification and drainage are necessary to improve the mortality associated with splenic abscess. Conservative management of splenic abscess with empiric antibiotic treatment and percutaneous drainage is an alternative to splenectomy, avoiding surgical complications and life-long susceptibility to encapsulated organisms.

Keywords: non-typhoidal salmonella, splenic abscess, splenectomy, percutaneous drainage
Volume 7 Issue $3-2019$

\author{
Arun Joseph Kattakayam,' Michelle R \\ Freeman, ${ }^{2}$ Sajin M Karakattu, ${ }^{3}$ Steffi Chinnu \\ Johns, ${ }^{4}$ Dima Youssef, ${ }^{5}$ Paolo Pino ${ }^{6}$ \\ 'Department of Internal Medicine, Norwalk Hospital, USA \\ ${ }^{2}$ Ross University Medical School, USA \\ ${ }^{3}$ Department of Internal Medicine, East Tennessee State \\ University, USA \\ ${ }^{4}$ Christian Medical College, India \\ ${ }^{5}$ Department of Infectious diseases, East Tennessee State \\ University, USA \\ ${ }^{6}$ Department of Infectious Disease, Norwalk Hospital, USA
}

Correspondence: Arun Joseph Kattakayam, MD, Department of Internal Medicine, Norwalk Hospital, 34 Maple Street Norwalk, CT, USA, Tel (203) 852-2000, Fax: (203) 899-5224, Email drjosephkattakayam@gmail.com

Received: May 21, 2019 | Published: June 24, 2019
Abbreviations: NTS, non-typhoidal salmonella; CT, computerized tomography; CRP, C-reactive protein; DMII, diabetes mellitus type II; HIV, human immunodeficiency virus; IV, intravenous

\section{Introduction}

Non-typhoidal salmonella (NTS) infections typically present with symptoms of gastroenteritis. Complications of NTS have an incidence of 49/100,000 cases that result in extra-intestinal manifestations including meningitis, osteomyelitis, bacteremia and in rare cases, abscesses. ${ }^{1-5}$ Risk factors for developing splenic abscesses include hematological malignancies, diabetes mellitus, sickle cell disease, endocarditis, trauma, intravenous drug use, immunosuppressive therapy and human immunodeficiency virus infection (HIV)., Splenic abscesses have been found to be associated most commonly with Salmonella, as well as Staphylococci, Streptococci, anaerobic organisms, Mycobacterium tuberculosis, Escherichia coli, and fungi. ${ }^{8-10}$ Diagnosis and visualization of splenic abscess is usually found via abdominal CT; however, ultrasound can also be used. ${ }^{11}$
Treatment of splenic abscess is traditionally divided into two groups of antibiotics with percutaneous drainage or antibiotics with splenectomy. If the splenic abscess is small or singular, conservative management with percutaneous drainage and antibiotics is preferred. However, in the case of large or multiple abscesses, splenectomy is preferred. ${ }^{12,13}$ Splenectomy is considered the gold standard treatment for splenic abscess. ${ }^{14}$ Currently, there is an ongoing debate as to whether or not splenectomy should still be the gold standard of care. Here, we provide a case where conservative management was used over splenectomy.

\section{Case presentation}

52-year-old Caucasian male with a past medical history of uncontrolled diabetes mellitus type 2 due to medication noncompliance, hypertension, and hypercholesterolemia, presented with two weeks of flu-like symptoms including fevers, night sweats, myalgias, and left lower back discomfort. He denied any symptoms of nausea, diarrhea or vomiting, as well as any history of travel outside the country. On 
physical exam, patient was febrile with a temperature of 38 . 6degree $\mathrm{C}$, tachycardic with a heart rate of 122 beats per minute, blood pressure of $122 / 80 \mathrm{mmHg}$, respiratory rate of 18 breaths per minute and oxygen saturation of $96 \%$ on room air. There was no tenderness to palpation on abdominal examination. Labs were significant for a white blood cell (WBC) count of $16.4 \mathrm{~K} / \mu \mathrm{L}$, hemoglobin A 1c of $12.2 \%$, alkaline phosphatase of $464 \mathrm{U} / \mathrm{L}$, alanine transaminase of $232 \mathrm{U} / \mathrm{L}$, aspartate amino transferase of $478 \mathrm{U} / \mathrm{L}$. CRP was elevated at $178.9 \mathrm{mg} / \mathrm{L}$. He tested negative for HIV and hepatitis A, B and C. Blood cultures were collected. Abdominal computerized tomography (CT) scan showed an enlarged spleen measuring $14 \mathrm{~cm}$ with a gas and fluid containing collection measuring approximately $6.2 \times 9.4 \times 5.6 \mathrm{~cm}$ suggestive of splenic abscess with adjacent free fluid and fat stranding abutting the diaphragm (Figure 1A). Patient was started on $2 \mathrm{~g}$ intravenous (IV) ceftriaxone daily. Blood cultures obtained on the day of admission subsequently grew Salmonella typhimurium. Transesophageal echocardiography was negative for vegetations. A percutaneous drain was placed the following day and the patient's symptoms significantly improved. CT scan on day six of IV antibiotics showed a decrease in size of the original splenic abscess, with identification of an additional smaller splenic abscess (Figure 1B). Patient was discharged on outpatient IV ceftriaxone for a total of six weeks, along with optimal glycemic control of his diabetes. A follow up CT scan after five weeks of treatment showed near resolution of the splenic abscesses (Figure 1C). The percutaneous drain was removed. Follow-up blood cultures at two and four weeks after completion of antibiotic treatment were negative.
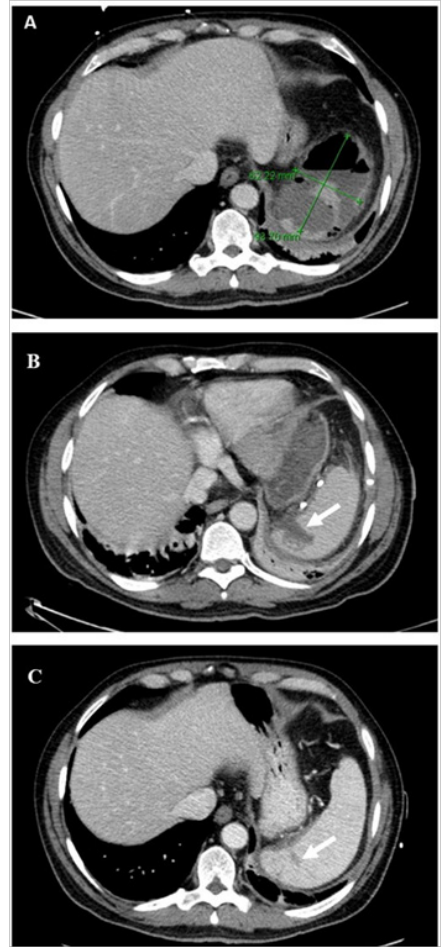

Figure I (A)Abdominal CT scans from day I showing splenomegaly measuring $14 \mathrm{~cm}$ with a gas and fluid containing collection measuring approximately $6.2 \times 9.4 \times 5.6 \mathrm{~cm}$ and adjacent free fluid and fat stranding abutting the diaphragm (marked with green arrows). (B) Day 6 of antibiotic therapy and drainage where the initial abscess has decreased in size to $3.0 \mathrm{~cm} \times 2.9 \mathrm{~cm}$. An additional $1.4 \times 2.9 \mathrm{~cm}$ perisplenic abscess was noted in the sub diaphragmatic space. (C) Follow up scan on day 24 of antibiotics showed thin crescent of fluid or granulation tissue deep to the splenic parenchyma and measuring up to $0.5 \mathrm{~cm}$ in thickness.

\section{Discussion}

Patients who have HIV, diabetes, malignancy, or are in an immunocompromised state are at a higher risk of infection. More specifically they are more susceptible to seeding of bacteria that can lead to complications such as an abscess, septic arthritis, meningitis or osteomyelitis. ${ }^{15}$ Non-typhoidal salmonella has multiple serovars that are prone to bacterial seeding in patients with these underlying comorbidities. ${ }^{16}$ There have been numerous case reports of NTS splenic abscesses in the literature; however management appears to be on a case by case basis. In previously healthy patients with splenic abscesses, there have been reports of successful antibiotic treatment with percutaneous drainage. ${ }^{17-19}$ There was also a report of a patient with uncontrolled diabetes who was treated conservatively with percutaneous drainage and antibiotics. ${ }^{20}$ Case reports for patients managed with antibiotics and splenectomy have also been published. ${ }^{18,21}$ There have been a few retrospective studies that compared antibiotic therapy with splenectomy versus percutaneous drainage, and have concluded that splenectomy was the definitive treatment. ${ }^{6,12}$ This conclusion is primarily drawn due to its success rate because of infection source control and probable selection bias, however splenectomy is not without risks. A well-known complication of splenectomy is the need for lifelong prophylactic protection from encapsulated microorganisms including Meningococcus, Pneumococcus and Haemophilus but the risks do not stop there. Studies have also found that splenectomy patients have lifelong complications including post-splenectomy septic syndrome in 2.5$13.5 \%$ of asplenic patients, increased risk of hemorrhagic and ischemic stroke, and chronic thromboembolic pulmonary hypertension. ${ }^{22-25}$ Targeted antibiotic intervention, with early detection and drainage may improve the outcome of patients with splenic abscess, but there are some promising proactive interventions on the horizon to prevent patients with comorbidities from developing a NTS complication. Efficacy in animal models for a live attenuated and subunit vaccines for multiple NTS serovars are currently in development. ${ }^{26}$

\section{Conclusion}

A rare potentially fatal complication of NTS infection is splenic abscess and should be considered in patients with left-sided abdominal pain and comorbidities such as diabetes or an immunocompromised state. Early identification and drainage are necessary to improve the mortality associated with splenic abscess. In the past, splenectomy was necessary. However, catheter drainage procedures have been successful. Here, we provide a case for conservative management of a large splenic abscess with additional smaller abscesses that resolved with empiric treatment and percutaneous drainage as an alternative treatment option to splenectomy, avoiding surgical complications and life-long susceptibility to multiple infections. Further research is needed to compare and balance the short term benefits, effectiveness, and lifelong complications between splenectomy and conservative management.

\section{Acknowledgements}

None.

\section{Conflict of interest}

The authors declare no conflict of interest.

\section{References}

1. Ao TT, Feasey NA, Gordon MA, et al. Global burden of invasive 
nontyphoidal Salmonella disease, 2010. Emerg Infect Dis. 2015;216:941-949.

2. Galanakis E, Bitsori M, Maraki S, et al. Invasive non-typhoida salmonellosis in immunocompetent infants and children. Int J Infect Dis. 2007;111:36-39.

3. Bitsori M, Galanakis E, Maraki S, et al. Invasive Salmonella virchow infection in childhood. Scand J Infect Dis. 2001;33:862-865.

4. Sirinavin S, Chiemchanya S, Vorachit M. Systemic nontyphoidal Salmonella infection in normal infants in Thailand. Pediatr Infect Dis J. 2001;20:581-587.

5. Kavaliotis J, Tsiaousi A, Papavasiliou D, et al. Non-typhoid Salmonella meningitis. Scand J Infect Dis. 1994;26:403-405.

6. Ooi LL, Leong SS. Splenic abscesses from 1987 to 1995. Am J Surg 1997; 174:87-93.

7. Guery R, Habibi A, Arlet JB, et al. Severe, non specific symptoms in nontyphoidal Salmonella infections in adult patients with sickle cell disease: a retrospective multicentre study. Infect Dis Lond. 2018;5011-12:822-830.

8. Galofre J, Moreno A, Mensa J, et al. Analysis of factors influencing the outcome and development of septic metastasis or relapse in Salmonella bacteremia. Clin Infect Dis. 1994;18:873-878.

9. Lee $\mathrm{CH}$, Leu HS, Hu TH, et al. Splenic abscess in southern Taiwan. $J$ Microbiol Immunol Infect. 2004;371:39-44.

10. Dhanoa A, Fatt QK. Non-typhoidal Salmonella bacteraemia epidemiology, clinical characteristics and its' association with severe immunosuppression. Ann Clin Microbiol Antimicrob. 2009;18:8-15.

11. Chang KC, Chuah SK, Changchien CS, et al. Clinical characteristics and prognostic factors of splenic abscess: a review of 67 cases in a single medical center of Taiwan. World J Gastroenterol. 2006;123:460-464.

12. Tung CC, Chen FC, Lo CJ. Splenic abscess: an easily overlooked disease? Am Surg. 2006;72:322-325.

13. De Greef E, Hoffman I, Topal B. Partial laparoscopic splenectomy for splenic abscess because of Salmonella infection: a case report. $J$ Pediat Surg. 2008;43:35-38.
14. Berendes TD, Keijman JM, te Velde LF, et al. Splenic abscesses caused by a reptile-associated salmonella infection. Dig Surg. 2007;24:397-399.

15. Acheson D, Hohmann EL. Nontyphoidal Salmonellosis. Clinical Infectious Diseases. 2001;321:263-269.

16. Zaidi E, Bachur R, Harper M. Non-typhi Salmonella bacteremia in children. Pediatr Infect Dis J. 1999;18:1073-1077.

17. Sillar JR, Bannan AM, Dahlenburg L. Splenic abscess complicating gastroenteritis due to Salmonella Virchow in an immunocompetent host. Med J. 2015;203(3):148-149.

18. Piplani S, Ramakrishna, Nandi B, et al. Two cases of salmonella splenic abscess. Med J Armed Forces India. 2006;621:77-78.

19. Bonares MJ, Ying T, Patel Y. Salmonella Thompson splenic abscess in a healthy female. BMJ Case Reports. 2017.

20. Manisa Sahu, Pradeep G Talwalkar. Splenic abscess due to salmonella paratyphi a: an incidental finding. Indian Journal of Medical Case Reports. 2015;4(2):20-22.

21. Cabadak H, Erbay A, Karaman K, et al. Splenic abscess due to Salmonella enteritidis. Infect Dis Rep. 2012;4(1):e4.

22. Shatney CH. Complications of splenectomy. Acta Anesthesiology Belg. 1987;38(4):333-339.

23. Lin JN, Lin CL, Lin MC, et al. Increased risk of hemorrhagic and ischemic strokes in patients with splenic injury and splenectomy: a Nationwide cohort study. Medicine Baltimore. 2015;94(35):e1458.

24. Jaïs X, Ioos V, Jardim C, et al. Splenectomy and chronic thromboembolic pulmonary hypertension. Thorax. 2005;60:1031-1034.

25. Sharon M Tennant, Calman A MacLennan, Raphael Simon, et al. Nontyphoidal salmonella disease: Current status of vaccine research and development. Vaccine. 2016;34(26):2907-2910.

26. Buzelé R, Barbier L, A. Sauvanet, B. Fanti. Medical complications following splenectomy. Journal de Chirurgie Viscérale. 2016 153(4):289299. 